\title{
TEMPO DE RETORNO DAS PRECIPITAÇÕES MÁXIMAS EM LAVRAS (MG) PELA DISTRIBUIÇÃO DE VALORES EXTREMOS DO TIPO I ${ }^{1}$
}

\author{
Maximum rainfall return period by extreme values type I distribution in Lavras, \\ Minas Gerais state, Brazil
}

Luiz Alberto Beijo², Joel Augusto Muniz ${ }^{3}$ Pedro Castro Neto ${ }^{4}$

\begin{abstract}
RESUMO
O conhecimento das precipitações máximas prováveis é de grande importância na elaboração de projetos agrícolas e de engenharia hidráulica. Tendo em vista esse fato, objetivou-se com este trabalho determinar as estimativas pontual e intervalar da precipitação diária máxima provável para os tempos de retorno de 2, 5, 10, 20, 30, 50 e 75 anos, para períodos de quinze dias, um mês e um ano na região de Lavras (MG), utilizando-se a distribuição de valores extremos do tipo I. Aplicou-se o método da máxima verossimilhança para a estimação dos parâmetros dessa distribuição, sendo os intervalos de confiança das estimativas de precipitações máximas prováveis obtidos aplicando-se técnica de reamostragem “Jackknife”. Pelos resultados, verificou-se que houve um bom ajuste da distribuição para os dados para a série anual, ao passo que na série mensal, nos meses de julho e agosto, ela não se ajustou. Para a série de quinze dias, o ajuste ocorreu apenas na estação chuvosa. Pelos resultados dos intervalos de confiança, pode-se adotar valores mais adequados de precipitação máxima no dimensionamento de obras hidráulicas na região de Lavras.
\end{abstract}

Termos para indexação: distribuição de valores extremos, intervalo de confiança, precipitação máxima, obras hidráulicas, técnica de reamostragem “Jackknife”.

\section{ABSTRACT}

The knowledge of the probable maximum rainfall is very important in the elaboration of agricultural and hydraulic engineering projects. Therefore this paper aimed to determine punctual and interval estimates of the probable maximum rainfall for return periods of 2, 5, 10, 20, 30, 50 and 75 years, at region of Lavras using the extreme values type I distribution, for periods of fifteen days, one month and one year. Maximum likelihood estimates of the parameters of the distribution were worked out. Jackknife Confidence Intervals for the estimates of probable maximum rainfall were calculated. A good fitting was observed for the annual series data; but not for the monthly, series data (specifically in july and august); and for the fifteen days series data no good model was found for the whole period, but just for the rainy season. One main conclusion is that the confidence intervals provided for the maximum rainfall values are the more appropriate to plan hydraulic works at Lavras region.

Index terms: extreme values type I distribution, confidence interval, maximum rainfall, hydraulic works, Jackknife re-sampling technique.

\section{(Recebido para publicação em $1^{\circ}$ de abril de 2004 e aprovado em 10 de março de 2005)}

\section{INTRODUÇÃO}

De acordo com Tucci (2001), a precipitação máxima é entendida como a ocorrência extrema, com duração, distribuição temporal e espacial críticas para uma área ou bacia hidrográfica. E esta pode atuar sobre a erosão do solo, inundações em áreas rurais e urbanas, obras hidráulicas, entre outros. A precipitação máxima provável (PMP) é definida como a maior coluna pluviométrica correspondente a uma duração fisicamente possível de ocorrer sobre uma determinada área em uma dada época do ano. O mesmo autor ainda comenta que a disponibilidade de longas séries de precipitações é, em geral, muito mais freqüente que a de vazão e que o estudo das precipitações máximas é um dos caminhos para se conhecer a vazão de enchente de uma bacia.

O conhecimento da precipitação diária máxima provável é importante para trabalhos de conservação do solo, estradas, barragens e drenagem, para cujo dimensionamento adequado é necessário conhecer ocorrências extremas. O fato de os projetos hidráulicos, em geral, serem concebidos considerando o custo mínimo, associado a um risco admissível de falha, requer a previsão de grandezas hidrológicas de grande magnitude, tais como máximas vazões ou precipitações que podem vir a ocorrer em certa localidade.

1. Parte da dissertação do primeiro autor apresentada à Universidade Federal de Lavras/UFLA - Caixa Postal 3037 - 37.200-000 - Lavras, MG.

2. Matemático, Ms. Doutorando em Agronomia - Estatística e Experimentação Agropecuária - Departamento de Ciências Exatas - UFLA Bolsista CAPES - luizbeijo@yahoo.com.br

3. Engenheiro Agrônomo - Dr. Professor Titular do departamento de Ciências Exatas da UFLA - Lavras,MG.

4. Engenheiro Agrônomo - Dr. Professor Titular do departamento de Engenharia da UFLA - Lavras, MG. 
Assim, as séries de máximos valores são empregadas para ajuste, segundo a lei probabilística que melhor descreva o processo, possibilitando extrapolações (VIEIRA et al., 1991).

A teoria de valores extremos, em especial a distribuição de valores extremos do tipo I, também conhecida como distribuição de Gumbel, distribuição tipo I de Fisher-Tippet, ou dupla exponencial, desenvolvida por Gumbel (1958), tem apresentado grande importância em vários campos da pesquisa, e tem sido aplicada com grande freqüência na análise estatística de variáveis ligadas a fenômenos meteorológicos, entre os quais a precipitação pluviométrica máxima.

Em estudo pioneiro sobre chuvas intensas no Brasil, Pfafstetter (1957) utilizou séries de valores máximos de precipitações de 98 estações pluviográficas distribuídas em diversas regiões do país, para a construção de curvas de intensidade-duraçãofreqüência, utilizando a distribuição de valores extremos do tipo I.

Estudando o ajustamento da distribuição de Gumbel a dados de precipitação pluviométrica máxima diária, coletados no período de 1914 a 1986 na Estação Climatológica Principal de Lavras, para o cálculo das precipitações máximas prováveis na estação chuvosa, Gomes et al. (1989) concluíram que os dados das precipitações máximas diárias ajustaram-se à distribuição em períodos de um ano e um mês, exceto para julho e em períodos de 10 e 15 dias, houve ajustamento do modelo somente na estação chuvosa.

Vasconcellos (1998) ajustou um modelo para descrever a chuva diária da região de Jaboticabal - SP, e obteve aplicando à distribuição de Gumbel as curvas intensidade-duração-freqüência, pela análise das precipitações máximas observadas, utilizando-se de registros históricos (1956 a 1995).

Vários outros autores têm utilizado a distribuição de valores extremos do tipo I em estudos de precipitações máximas, seja para determinar a precipitação máxima provável ou o período de recorrência de chuvas máximas ou as curvas intensidade-duração-freqüência, destacandose Back (1996), Beltrame et al. (1991), Eltz et al. (1992), Leopoldo et al. (1984), Nobukuni (2000), Vieira et al. (1994) e Pinto (1999), entre outros.

Tendo em vista a grande importância do conhecimento das possíveis precipitações diárias máximas, realizou-se este trabalho com o objetivo de analisar o ajustamento da distribuição de valores extremos do tipo I aos dados agrupados em períodos de quinze dias, um mês e um ano; e estimar a precipitação pluvial diária máxima provável com seus respectivos intervalos de confiança para os tempos de retorno de 2, $5,10,20,30,50$ e 75 anos, nos períodos de quinze dias, mensal e anual, na região de Lavras-MG.

\section{MATERIAL E MÉTODOS}

Os dados utilizados no presente estudo foram obtidos dos registros pluviométricos da Estação Climatológica Principal de Lavras-MG, situada no campus da Universidade Federal de Lavras, em Lavras, Minas Gerais, em convênio com o Instituto Nacional de Meteorologia (INMET). A Estação Climatológica está situada nas coordenadas geográficas de $21^{0} 14$ ' de latitude Sul, $45^{0} 00^{\prime}$ de longitude Oeste de Greenwich e 918,8m de altitude média (BRASIL, 1992). Segundo a classificação de Koppen (VIANELLO \& ALVES, 1991), o clima da região é do tipo Cwa. Em Brasil (1992), encontram-se valores totais e médios mensais no período de 1961 a 1990, de 23,4 mm no mês mais seco, 295,8 mm no mês mais chuvoso e precipitação total anual de $1529,7 \mathrm{~mm}$.

As observações referem-se às precipitações pluviais diárias expressas em altura de lâmina d'água (mm), referentes a 90 anos de observação do período compreendido entre janeiro de 1914 a dezembro de 2003, sendo eliminados os períodos dos anos que apresentaram falhas nos registros, reduzindo em alguns casos para 87 anos, permanecendo dentro da faixa recomendada pela Organização Mundial de Meteorologia, que é, segundo Pinto (1999), de 30 anos.

Para o estudo do ajustamento da distribuição de valores extremos do tipo I aos dados, esses foram agrupados em períodos de quinze dias, mensal e anual. Para o período de quinze dias, o ano foi dividido em 24 períodos discretos respectivamente, sendo cada mês 2 períodos. Nos meses com 31 dias, o último período para o caso ficou com 16 dias; no caso do mês de fevereiro, esse período ficou com um ou dois dias a menos. Para o período de um mês, foram considerados os 12 meses do ano, e o ano foi considerado um período. Extraindo-se a máxima precipitação pluvial diária observada de cada período formaram-se os vetores de precipitações máximas. Com esses vetores, foram determinadas as estimativas dos parâmetros da distribuição de valores extremos do tipo I pelo método da máxima verossimilhança e, utilizando-se essas estimativas dos parâmetros, calculou-se a precipitação pluvial diária máxima provável para os tempos de retorno de $2,5,10,20,30,50$ e 75 anos para cada um dos períodos estudados e seus respectivos intervalos de 
confiança via técnica de reamostragem “Jackknife”, e para avaliar a adeqüacidade da distribuição de valores extremos do tipo I, em todos os períodos estudados, foi utilizado o teste Kolmogorov-Smirnov, a um nível de 5\% de significância, de acordo com Campos (1979).

A função de densidade de probabilidades (f.d.p) da distribuição de valores extremos do tipo I, da variável aleatória $X$, associada a valores máximos, é dada por:

$$
f(x ; \beta, \alpha)=\frac{1}{\alpha} \exp \left\{-\left(\frac{x-\beta}{\alpha}\right)-\exp \left[-\left(\frac{x-\beta}{\alpha}\right)\right]\right\},
$$

em que $X$ é a variável aleatória associada a valores máximos do período e $-\infty<x<\infty, \quad \beta$ é denominado parâmetro de posição e $-\infty<\beta<\infty$, $\alpha$ o parâmetro de escala e $\alpha>0$.

A função de distribuição acumulada da variável aleatória $\mathrm{X}$, para valores extremos máximos, é dada por:

$$
F(x ; \beta, \alpha)=\int_{0}^{x} f(x ; \beta, \alpha) d x=\exp \left\{-\exp \left[-\left(\frac{x-\beta}{\alpha}\right)\right]\right\}
$$

A probabilidade $(P)$ de que ocorra uma precipitação pluvial máxima maior que um certo valor $X$ é dada por:

$$
P=1-F(x ; \beta, \alpha)=1-\exp \left\{-\exp \left[-\left(\frac{x-\beta}{\alpha}\right)\right]\right\},
$$

em que $X$ é a precipitação máxima do período e $0<x<\infty$.

De acordo com Lowery \& Nash (1970), a precipitação pluvial máxima provável para um determinado tempo de retorno $T$ pode ser determinada pela seguinte expressão:

$$
X=\beta-\alpha \ln \left[\ln \left(\frac{T}{T-1}\right)\right]
$$

Segundo Haan (1977), os estimadores da máxima verossimilhança (EMV) para os parâmetros $\beta$ e $\alpha$, obtidos a partir da f.d.p conjunta da distribuição de valores extremos do tipo I, são, respectivamente,

$$
\begin{aligned}
& \hat{\beta}=-\hat{\alpha} \ln \left[\frac{1}{n} \sum_{i=1}^{n} \exp \left(\frac{-x_{i}}{\hat{\alpha}}\right)\right], \\
& \hat{\alpha}=\frac{1}{n} \sum_{i=1}^{n} x_{i}-\frac{\sum_{i=1}^{n} x_{i} \exp \left(\frac{-x_{i}}{\hat{\alpha}}\right)}{\sum_{i=1}^{n} \exp \left(\frac{-x_{i}}{\hat{\alpha}}\right)},
\end{aligned}
$$

em que $\ln$ é o operador do logaritmo neperiano.

Percebe-se pelos valores de $\hat{\alpha}$ e $\hat{\beta}$ que os estimadores (EMV) não possuem expressões explícitas, exigindo cálculos numéricos iterativos e, portanto, deve-se primeiro determinar $\hat{\alpha}$ como solução de (6), e substituir esse valor em (5) para obter $\hat{\beta}$. Devido ao fato de $\hat{\alpha}$ não poder ser obtido analiticamente, deve-se determinar por aproximação numérica, e conforme Vivanco (1994), o procedimento iterativo de NewtonRaphson é recomendável nesse caso. Para a estimação de $\beta$ e $\alpha$, foi usado o programa elaborado no software Statistical Analysis System (SAS ${ }^{\circledR}$ ) 6.12, usando o PROC IML - Procedimento "Interactive Matrix Language ", (SAS INSTITUTE, 1990).

A deficiência observada nas estimativas pontuais, no presente caso de precipitação máxima, é o fato de que essas não expressam a confiança que se possa ter acerca da maior ou menor diferença entre a estimativa de precipitação máxima e seu valor real. De acordo Mood et al. (1974), a inferência pode ser complementada sempre que possível, com pressuposições acerca de probabilidades de esse parâmetro (precipitação máxima) estar próximo ou não de suas estimativas pontuais. Isso pode ser feito 
mediante a construção de intervalos de confiança com probabilidade conhecida de que o valor paramétrico esteja ali contido.

Com o objetivo de se obter estimativas mais confiáveis de precipitação máxima provável, foram calculados os seus respectivos intervalos de confiança aplicando-se a técnica de reamostragem "Jackknife”, de acordo com Manly (1998). A utilização dessa técnica de reamostragem foi de suma importância para obtenção dos intervalos, pois é sabido que a estimação de intervalos de confiança para a expressão (4) não apresenta uma expressão analítica.

Determinou-se também a proporção dos meses em que ocorreu a precipitação máxima no período de um ano pela expressão:

$$
P_{i}=\frac{j_{i}}{n}
$$

em que, $j_{i}$ é o número de vezes que a precipitação máxima anual ocorreu no i-ésimo mês, e $n$ é o tamanho da série anual de precipitação diária máxima.

\section{RESULTADOS E DISCUSSÃO}

A série de precipitações diárias máximas mensais relativas aos anos estudados é apresentada graficamente na Figura 1.

Na Figura 1, nota-se que poucas precipitações diárias máximas mensais (acumuladas em 24 horas) superaram $100 \mathrm{~mm}$, destacando-se a observação 937, que corresponde à precipitação diária ocorrida no dia 23 de janeiro de 1992, que foi de 202 milímetros de lâmina d'água. As precipitações diárias máximas mensais que superaram 100 milímetros de lâmina d’água e o respectivo mês e ano em que ocorreram são apresentadas no Quadro 1.

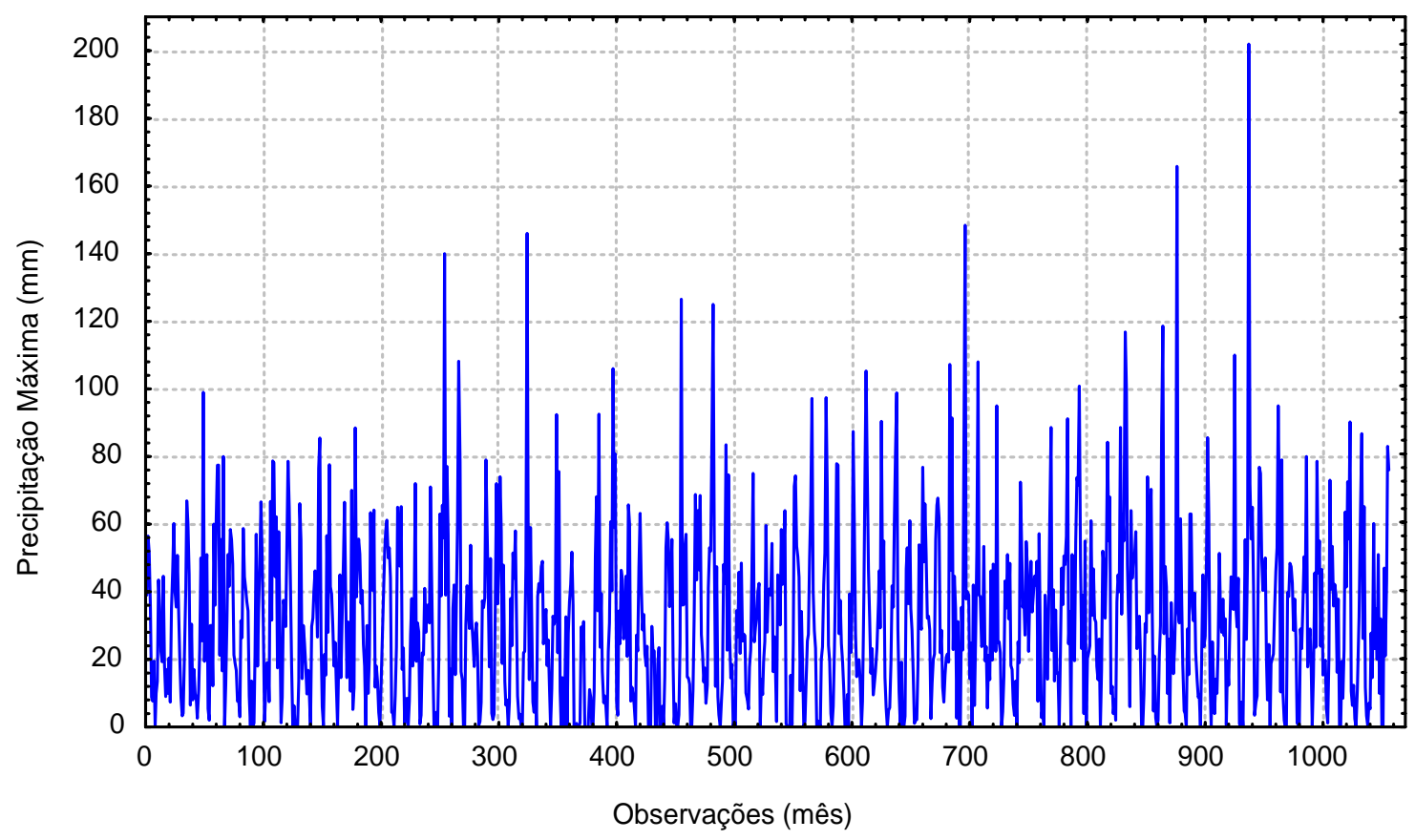

FIGURA 1 - Representação gráfica da série de precipitações diárias máximas mensais (em mm) no município de Lavras - MG, no período de janeiro de 1914 a dezembro de 2003. 
QUADRO 1 - Precipitações diárias máximas mensais que superaram 100 mm, no município de Lavras - MG, ocorridas entre janeiro de 1914 e dezembro de 2003.

\begin{tabular}{|l|c|l|c|}
\hline Período de Ocorrência & Precipitação (mm) & Período de Ocorrência & Precipitação (mm) \\
\hline Janeiro de 1992 & 202 & Abril de 1983 & 116,8 \\
Dezembro de 1986 & 165,8 & Janeiro de 1991 & 110 \\
Dezembro de 1971 & 148,4 & Fevereiro de 1936 & 108,2 \\
Dezembro de 1940 & 146 & Novembro de 1972 & 108 \\
Fevereiro de 1935 & 140 & Novembro de 1970 & 107,2 \\
Novembro de 1951 & 126,5 & Janeiro de 1947 & 106 \\
Fevereiro de 1954 & 125 & Dezembro de 1964 & 105,3 \\
Dezembro de 1985 & 118,6 & Janeiro de 1980 & 100,8 \\
\hline
\end{tabular}

Analisando-se o Quadro 1, pode-se verificar que a maioria das precipitações máximas que superaram 100 mm ocorreram em janeiro e dezembro.

Os dados de precipitação diária máxima agrupados em período de um ano ajustaram-se à distribuição de valores extremos do tipo I. Quando o período de observação foi de um mês, observou-se o ajustamento, excetuando-se julho e agosto. Para períodos de 15 dias, o ajustamento se deu em toda a estação chuvosa, não havendo ajustamento para as quinzenas de maio, junho, julho, agosto e primeira quinzena de setembro, conforme avaliado pelo teste Kolmogorov-Smirnov. Resultados semelhantes foram obtidos por Gomes et al. (1989), excetuando-se agosto do período de um mês, e a primeira quinzena de maio, em que a série de precipitações máximas diárias estudada pelos autores se ajustou à distribuição de valores extremos do tipo I, fato esse que pode ser explicado pela pouca ocorrência de precipitação nesses períodos após 1986.

É importante ressaltar que aplicou-se a distribuição de valores extremos do tipo I para obter as estimativas de precipitações máximas apenas para os períodos em que houve o ajuste.

Na Tabela 1, observando-se as médias mensais das precipitações máximas diárias apresentadas, pode-se verificar que os meses de janeiro e dezembro foram os que apresentaram maiores valores, $(57 \mathrm{~mm}$ e $55 \mathrm{~mm}$ ) respectivamente. Junho teve a menor média (12 mm), sendo a média das precipitações máximas diárias para o período anual de $80 \mathrm{~mm}$. Valores parecidos foram observados por Beijo et al. (2003), que analisando as precipitações diárias máximas de
Jaboticabal (SP), notaram que os meses de janeiro e dezembro apresentaram ambos a média $55 \mathrm{~mm}$, e o período anual média de $79 \mathrm{~mm}$. Isso demonstra que a distribuição das precipitações máximas nas duas regiões são parecidas.

A proporção dos meses em que ocorreu a precipitação máxima anual variou ao longo dos meses mais chuvosos, e os meses de novembro, dezembro, janeiro e fevereiro apresentaram uma proporção de 0,$17 ; 0,21 ; 0,26$ e 0,17 , respectivamente, ou seja, $81 \%$ das precipitações máximas no período de um ano ocorreram nesses meses, enquanto nos meses mais secos não ocorreu nenhuma precipitação máxima anual (Tabela 1). Essa informação pode ser importante para o planejamento de obras, principalmente de projetos agrícolas e de engenharia hidráulica, quando se relaciona a início e execução das obras.

Analisado-se as Tabelas 1 e 2, pode-se verificar que as estimativas dos parâmetros $\beta$ e $\alpha$ foram menores nos meses mais secos em que houve ajuste (maio, junho e setembro) e maiores nos meses mais chuvosos. Mesmo não sendo a mesma região, resultados semelhantes foram observados por Beijo et al. (2003), que analisaram as precipitações diárias máximas de Jaboticabal (SP) utilizando a distribuição de valores extremos do tipo I.

Em relação às médias quinzenais das precipitações máximas diárias apresentadas na Tabela 2, verificou-se que a segunda quinzena de dezembro, a primeira e a segunda quinzenas de janeiro foram as que apresentaram maiores valores, (48 mm, $44 \mathrm{~mm}$ e 48 $\mathrm{mm}$ ), respectivamente. 
TABELA 1 - Estimativas dos parâmetros $\beta$ e $\alpha$ da distribuição de valores extremos do tipo I, da probabilidade de ocorrer a precipitação máxima anual $P$ e da média das precipitações diárias máximas $(\mathrm{mm})$ nos diferentes meses, em Lavras - MG.

\begin{tabular}{|c|c|c|c|c|c|c|c|c|c|}
\hline Período & $\hat{\beta}$ & $\hat{\alpha}$ & $P$ & Média & Período & $\hat{\beta}$ & $\hat{\alpha}$ & $P$ & Média \\
\hline Jan. & 45,48 & 19,09 & 0,26 & 57 & Fev. & 36,52 & 19,06 & 0,17 & 47 \\
\hline Mar. & 34,11 & 15,28 & 0,07 & 44 & Abr. & 17,65 & 12,50 & 0,04 & 25 \\
\hline Mai. & 11,65 & 11,74 & 0,00 & 19 & Jun. & 6,60 & 7,94 & 0,00 & 12 \\
\hline Set. & 15,13 & 11,72 & 0,01 & 22 & Out. & 28,73 & 12,73 & 0,08 & 36 \\
\hline Nov. & 37,85 & 16,04 & 0,17 & 48 & Dez. & 44,94 & 15,06 & 0,21 & 55 \\
\hline Ano & 67,63 & 20,75 & 1,00 & 80 & & & & & \\
\hline
\end{tabular}

TABELA 2 - Estimativas dos parâmetros $\beta$ e $\alpha$ da distribuição de valores extremos do tipo I e da média das precipitações diárias máximas (mm) nas diferentes quinzenas, em Lavras - MG.

\begin{tabular}{|c|c|c|c|c|c|c|c|}
\hline Período & $\hat{\beta}$ & $\hat{\alpha}$ & Média & Período & $\hat{\beta}$ & $\hat{\alpha}$ & Média \\
\hline Jan. 1Q & 33,47 & 18,06 & 44 & Jan. 2Q & 32,42 & 20,90 & 45 \\
\hline Fev. 1Q & 27,35 & 17,12 & 37 & Fev. 2Q & 25,81 & 17,10 & 36 \\
\hline Mar. 1Q & 27,74 & 16,01 & 37 & Mar. 2Q & 21,30 & 14,48 & 30 \\
\hline Abr. 1Q & 13,05 & 12,29 & 21 & Abr. 2Q & 7,38 & 8,41 & 13 \\
\hline Set. 2Q & 9,68 & 9,77 & 15 & Out. 1Q & 17,29 & 14,22 & 26 \\
\hline Out. 2Q & 21,18 & 12,49 & 28 & Nov. 1Q & 27,25 & 15,29 & 36 \\
\hline Nov. 2Q & 28,92 & 15,33 & 38 & Dez. 1Q & 32,10 & 14,10 & 40 \\
\hline Dez. 2Q & 36,98 & 16,50 & 48 & & & & \\
\hline
\end{tabular}

* Ao nível de 95\% de confiança; 1Q: Primeira quinzena do mês referente; 2Q: Segunda quinzena do mês referente.

As estimativas das precipitações diárias máximas prováveis para os tempos de retorno de 2, 5, 10, 20, 30, 50, e 75 anos, e seus respectivos intervalos de confiança determinadas para os períodos mensal e anual são apresentados nas Tabelas 3 , 4 e 5.

Na Tabela 3, escolhendo 50 anos como o tempo de retorno adequado de ocorrer uma determinada precipitação pluvial máxima diária para o período anual, uma interpretação das informações pode ser feita do seguinte modo: espera-se que, em um tempo médio de
50 anos, o valor da precipitação diária máxima no ano seja superior a $148 \mathrm{~mm}$, tendo um intervalo de $95 \%$ de confiança, com limites superior e inferior $(131 \mathrm{~mm}$; $164 \mathrm{~mm}$ ), respectivamente.

Análises semelhantes podem ser feitas para todos os períodos nas Tabelas 3, 4 e 5 .

Pode-se notar nas Tabelas 3, 4 e 5 que as estimativas das precipitações pluviais máximas diária aumentam à medida que se aumenta o tempo de retorno. 
Tempo de retorno das precipitações máximas em...

TABELA 3 - Estimativas das precipitações pluviais máximas diária $(\mathrm{mm})$ na região de Lavras-MG, para diferentes tempos de retorno (em anos) obtidas via distribuição de valores extremos tipo I e limites inferior (LI) e superior (LS) de seus respectivos intervalos de confiança*, para o período mensal e anual.

\begin{tabular}{|c|c|c|c|c|c|c|c|c|c|c|c|}
\hline \multicolumn{4}{|c|}{ Janeiro } & \multicolumn{4}{|c|}{ Fevereiro } & \multicolumn{4}{|c|}{ Março } \\
\hline Tempo & Precip. & LI & LS & Tempo & Precip. & LI & LS & Tempo & Precip. & LI & LS \\
\hline 2 & 53 & 48 & 57 & 2 & 43 & 38 & 48 & 2 & 41 & 37 & 45 \\
\hline 5 & 74 & 66 & 82 & 5 & 65 & 57 & 72 & 5 & 58 & 52 & 64 \\
\hline 10 & 88 & 78 & 99 & 10 & 79 & 69 & 89 & 10 & 69 & 62 & 77 \\
\hline 20 & 102 & 89 & 115 & 20 & 93 & 81 & 104 & 20 & 80 & 72 & 89 \\
\hline 30 & 110 & 96 & 124 & 30 & 101 & 88 & 114 & 30 & 87 & 77 & 96 \\
\hline 50 & 120 & 104 & 136 & 50 & 110 & 96 & 125 & 50 & 95 & 84 & 105 \\
\hline 75 & 128 & 110 & 145 & 75 & 118 & 102 & 134 & 75 & 101 & 90 & 112 \\
\hline \multicolumn{4}{|c|}{ Abril } & \multicolumn{4}{|c|}{ Maio } & \multicolumn{4}{|c|}{ Junho } \\
\hline Tempo & Precip. & LI & LS & Tempo & Precip. & LI & LS & Tempo & Precip. & LI & LS \\
\hline 2 & 22 & 19 & 25 & 2 & 16 & 13 & 19 & 2 & 9 & 7 & 12 \\
\hline 5 & 36 & 31 & 42 & 5 & 29 & 24 & 34 & 5 & 18 & 14 & 22 \\
\hline 10 & 46 & 38 & 53 & 10 & 38 & 31 & 45 & 10 & 24 & 19 & 30 \\
\hline 20 & 55 & 46 & 64 & 20 & 46 & 38 & 55 & 20 & 30 & 23 & 37 \\
\hline 30 & 60 & 50 & 70 & 30 & 51 & 42 & 60 & 30 & 33 & 26 & 41 \\
\hline 50 & 66 & 55 & 77 & 50 & 57 & 47 & 68 & 50 & 37 & 29 & 46 \\
\hline 75 & 71 & 59 & 83 & 75 & 62 & 51 & 73 & 75 & 41 & 32 & 50 \\
\hline \multicolumn{4}{|c|}{ Setembro } & \multicolumn{4}{|c|}{ Outubro } & \multicolumn{4}{|c|}{ Novembro } \\
\hline Tempo & Precip. & LI & LS & Tempo & Precip. & LI & LS & Tempo & Precip. & LI & LS \\
\hline 2 & 19 & 17 & 22 & 2 & 33 & 30 & 36 & 2 & 44 & 40 & 48 \\
\hline 5 & 33 & 29 & 36 & 5 & 48 & 43 & 52 & 5 & 62 & 55 & 68 \\
\hline 10 & 41 & 36 & 46 & 10 & 57 & 51 & 63 & 10 & 74 & 65 & 82 \\
\hline 20 & 50 & 44 & 55 & 20 & 66 & 59 & 74 & 20 & 85 & 75 & 95 \\
\hline 30 & 54 & 48 & 61 & 30 & 71 & 63 & 80 & 30 & 92 & 81 & 103 \\
\hline 50 & 61 & 53 & 68 & 50 & 78 & 69 & 87 & 50 & 100 & 87 & 113 \\
\hline 75 & 65 & 58 & 73 & 75 & 83 & 74 & 93 & 75 & 107 & 93 & 120 \\
\hline \multicolumn{4}{|c|}{ Dezembro } & & & & & \multicolumn{4}{|c|}{ Ano } \\
\hline Tempo & Precip. & LI & LS & & & & & Tempo & Precip. & LI & LS \\
\hline 2 & 50 & 46 & 54 & & & & & 2 & 75 & 70 & 80 \\
\hline 5 & 67 & 60 & 75 & & & & & 5 & 98 & 90 & 107 \\
\hline 10 & 79 & 69 & 88 & & & & & 10 & 114 & 103 & 125 \\
\hline 20 & 89 & 77 & 101 & & & & & 20 & 129 & 115 & 142 \\
\hline 30 & 96 & 82 & 109 & & & & & 30 & 137 & 123 & 152 \\
\hline 50 & 103 & 88 & 118 & & & & & 50 & 148 & 131 & 164 \\
\hline 75 & 109 & 93 & 126 & & & & & 75 & 156 & 138 & 174 \\
\hline
\end{tabular}

* Ao nível de 95\% de confiança. 
TABELA 4 - Estimativas das precipitações pluviais máximas diária $(\mathrm{mm})$ na região de Lavras-MG, para diferentes tempos de retorno (em anos) obtidas via distribuição de valores extremos tipo I e limites inferior (LI) e superior (LS) de seus respectivos intervalos de confiança* para o período de quinze dias dos meses de janeiro a abril.

\begin{tabular}{|c|c|c|c|c|c|c|c|c|c|c|c|}
\hline \multicolumn{4}{|c|}{ Janeiro 1 Q } & \multicolumn{4}{|c|}{ Janeiro 2 Q } & \multicolumn{4}{|c|}{ Fevereiro $1 \mathrm{Q}$} \\
\hline $\begin{array}{l}\text { Tempo } \\
\text { retorno }\end{array}$ & $\begin{array}{c}\text { Precip. } \\
\text { (mm) }\end{array}$ & LI & LS & $\begin{array}{l}\text { Tempo } \\
\text { retorno }\end{array}$ & $\begin{array}{c}\text { Precip. } \\
\text { (mm) }\end{array}$ & LI & LS & $\begin{array}{l}\text { Tempo } \\
\text { retorno }\end{array}$ & $\begin{array}{l}\text { Precip. } \\
\text { (mm) }\end{array}$ & LI & LS \\
\hline 2 & 40 & 36 & 45 & 2 & 40 & 35 & 46 & 2 & 33 & 29 & 38 \\
\hline 5 & 61 & 54 & 68 & 5 & 64 & 56 & 72 & 5 & 53 & 46 & 60 \\
\hline 10 & 74 & 65 & 83 & 10 & 80 & 70 & 90 & 10 & 66 & 57 & 76 \\
\hline 20 & 87 & 76 & 98 & 20 & 95 & 82 & 107 & 20 & 79 & 67 & 90 \\
\hline 30 & 95 & 83 & 107 & 30 & 103 & 90 & 117 & 30 & 86 & 74 & 99 \\
\hline 50 & 104 & 90 & 118 & 50 & 114 & 99 & 130 & 50 & 95 & 81 & 109 \\
\hline 75 & 112 & 97 & 126 & 75 & 123 & 106 & 140 & 75 & 102 & 87 & 117 \\
\hline \multicolumn{4}{|c|}{ Fevereiro $2 \mathrm{Q}$} & \multicolumn{4}{|c|}{ Março 1 Q } & \multicolumn{4}{|c|}{ Março 2 Q } \\
\hline $\begin{array}{l}\text { Tempo } \\
\text { retorno }\end{array}$ & $\begin{array}{l}\text { Precip. } \\
\text { (mm) }\end{array}$ & LI & LS & $\begin{array}{l}\text { Tempo } \\
\text { retorno }\end{array}$ & $\begin{array}{c}\text { Precip. } \\
\text { (mm) }\end{array}$ & LI & LS & $\begin{array}{l}\text { Tempo } \\
\text { retorno }\end{array}$ & $\begin{array}{l}\text { Precip. } \\
\text { (mm) }\end{array}$ & LI & LS \\
\hline 2 & 32 & 28 & 36 & 2 & 34 & 30 & 38 & 2 & 27 & 23 & 30 \\
\hline 5 & 51 & 45 & 58 & 5 & 52 & 47 & 57 & 5 & 43 & 37 & 49 \\
\hline 10 & 64 & 56 & 72 & 10 & 64 & 57 & 71 & 10 & 54 & 46 & 61 \\
\hline 20 & 76 & 66 & 86 & 20 & 76 & 67 & 84 & 20 & 64 & 55 & 73 \\
\hline 30 & 83 & 72 & 94 & 30 & 82 & 73 & 91 & 30 & 70 & 60 & 80 \\
\hline 50 & 92 & 79 & 105 & 50 & 90 & 80 & 101 & 50 & 77 & 66 & 89 \\
\hline 75 & 99 & 85 & 113 & 75 & 97 & 86 & 108 & 75 & 83 & 71 & 96 \\
\hline \multicolumn{4}{|c|}{ Abril $1 \mathrm{Q}$} & \multicolumn{4}{|c|}{ Abril 2 Q } & & & & \\
\hline $\begin{array}{l}\text { Tempo } \\
\text { retorno }\end{array}$ & $\begin{array}{c}\text { Precip. } \\
\text { (mm) }\end{array}$ & LI & LS & $\begin{array}{l}\text { Tempo } \\
\text { retorno }\end{array}$ & $\begin{array}{l}\text { Precip. } \\
\text { (mm) }\end{array}$ & LI & LS & & & & \\
\hline 2 & 18 & 14 & 21 & 2 & 10 & 8 & 13 & & & & \\
\hline 5 & 31 & 26 & 37 & 5 & 20 & 16 & 24 & & & & \\
\hline 10 & 41 & 33 & 48 & 10 & 26 & 21 & 32 & & & & \\
\hline 20 & 49 & 40 & 59 & 20 & 32 & 26 & 39 & & & & \\
\hline 30 & 55 & 44 & 65 & 30 & 36 & 28 & 43 & & & & \\
\hline 50 & 61 & 49 & 73 & 50 & 40 & 32 & 48 & & & & \\
\hline 75 & 66 & 53 & 79 & 75 & 43 & 34 & 52 & & & & \\
\hline
\end{tabular}

* Ao nível de 95\% de confiança; 1Q: Primeira quinzena do mês referente; 2Q: Segunda quinzena do mês referente, 
Uma observação importante a ser feita nas Tabelas 3, 4 e 5 se refere aos intervalos de confiança obtidos para cada estimativa de precipitação máxima. Como em análises de precipitações máximas sempre se está interessado no evento extremo máximo, sugere-se nesse caso utilizar o limite superior do intervalo como valor de referência. Um exemplo a ser dado é a chuva ocorrida em janeiro de 1991 (110 mm), conforme apresentado no Quadro 1. Em seu trabalho, Gomes et al. (1989) calcularam a precipitação pluviométrica máxima esperada para vários níveis de probabilidade, e obtiveram para o nível de 5\% no mês de janeiro, que corresponde a um período de retorno de 20 anos, o valor estimado de $99 \mathrm{~mm}$, ou seja, esperava-se que a precipitação máxima de $99 \mathrm{~mm}$ seria superada com um tempo médio de retorno de 20 anos. Porém, em um período de tempo bem menor (aproximadamente 5 anos), ocorreu uma precipitação de $110 \mathrm{~mm}$, e pode-se concluir que houve uma subestimação da quantidade de precipitação máxima, o que não aconteceria se tivesse sido calculado o intervalo de confiança para a precipitação máxima esperada e adotasse, nesse caso, o limite superior do intervalo como referência (que seria, para esse caso, de $113 \mathrm{~mm}$ ).

TABELA 5 - Estimativas das precipitações pluviais máximas diárias (mm) na região de Lavras-MG, para diferentes tempos de retorno (em anos) obtidas via distribuição de valores extremos tipo I e limites inferior (LI) e superior (LS) de seus respectivos intervalos de confiança* para o período de quinze dias dos meses de setembro a dezembro.

\begin{tabular}{|c|c|c|c|c|c|c|c|c|c|c|c|}
\hline \multicolumn{4}{|c|}{ Setembro $2 \mathrm{Q}$} & \multicolumn{4}{|c|}{ Outubro $1 \mathrm{Q}$} & \multicolumn{4}{|c|}{ Outubro 2 Q } \\
\hline Tempo & Precip. & LI & LS & Tempo & Precip. & $\mathrm{LI}$ & LS & Tempo & Precip. & LI & LS \\
\hline 2 & 13 & 11 & 16 & 2 & 22 & 19 & 26 & 2 & 26 & 23 & 29 \\
\hline 5 & 24 & 20 & 28 & 5 & 38 & 33 & 44 & 5 & 40 & 35 & 44 \\
\hline 10 & 31 & 27 & 36 & 10 & 49 & 42 & 56 & 10 & 49 & 43 & 55 \\
\hline 20 & 38 & 33 & 44 & 20 & 59 & 51 & 68 & 20 & 58 & 51 & 65 \\
\hline 30 & 42 & 36 & 49 & 30 & 65 & 56 & 74 & 30 & 63 & 56 & 71 \\
\hline 50 & 48 & 40 & 55 & 50 & 72 & 62 & 83 & 50 & 70 & 61 & 78 \\
\hline 75 & 51 & 44 & 59 & 75 & 78 & 67 & 89 & 75 & 75 & 65 & 84 \\
\hline \multicolumn{4}{|c|}{ Novembro $1 \mathrm{Q}$} & \multicolumn{4}{|c|}{ Novembro $2 \mathrm{Q}$} & \multicolumn{4}{|c|}{ Dezembro $1 \mathrm{Q}$} \\
\hline Tempo & Precip. & LI & LS & Tempo & Precip. & LI & LS & Tempo & Precip. & LI & LS \\
\hline 2 & 33 & 29 & 36 & 2 & 35 & 31 & 39 & 2 & 37 & 34 & 41 \\
\hline 5 & 50 & 44 & 56 & 5 & 52 & 45 & 59 & 5 & 53 & 49 & 58 \\
\hline 10 & 61 & 54 & 69 & 10 & 63 & 55 & 72 & 10 & 64 & 58 & 69 \\
\hline 20 & 72 & 63 & 81 & 20 & 74 & 64 & 85 & 20 & 74 & 67 & 81 \\
\hline 30 & 79 & 68 & 89 & 30 & 81 & 69 & 92 & 30 & 80 & 73 & 87 \\
\hline 50 & 87 & 75 & 98 & 50 & 89 & 76 & 102 & 50 & 87 & 79 & 95 \\
\hline 75 & 93 & 80 & 105 & 75 & 95 & 81 & 109 & 75 & 93 & 84 & 102 \\
\hline \multicolumn{4}{|c|}{ Dezembro $2 \mathrm{Q}$} & & & & & & & & \\
\hline Tempo & Precip. & LI & LS & & & & & & & & \\
\hline 2 & 43 & 39 & 48 & & & & & & & & \\
\hline 5 & 62 & 53 & 70 & & & & & & & & \\
\hline 10 & 74 & 63 & 85 & & & & & & & & \\
\hline 20 & 86 & 72 & 99 & & & & & & & & \\
\hline 30 & 93 & 78 & 107 & & & & & & & & \\
\hline 50 & 101 & 84 & 118 & & & & & & & & \\
\hline 75 & 108 & 89 & 126 & & & & & & & & \\
\hline
\end{tabular}




\section{CONCLUSÕES}

Cerca de $81 \%$ das precipitações máximas do ano ocorreram nos meses de novembro, dezembro, janeiro e fevereiro.

Os dados de precipitação diária máxima agrupados nos períodos mensal e anual ajustaram-se à distribuição de valores extremos tipo I, excetuando-se os meses de julho e agosto. Já para os períodos de quinze dias, houve ajuste somente para a estação chuvosa.

As estimativas intervalares fornecem valores mais seguros de precipitações diárias máximas prováveis, e podem ser usadas para auxiliar no planejamento de obras de engenharia hidráulica e agrícola na região de Lavras (MG).

\section{REFERÊNCIAS BIBLIOGRÁFICAS}

BACK, Á. J. Análise das máximas intensidades de chuva para a região de Urussanga - SC. In: CONGRESSO NACIONAL DE ENGENHARIA DE AGRIMENSURA, 7., 1996, Salvador, BA. Anais... Salvador: FENEA, 1996. p. 75-80.

BEIJO, L. A.; MUNIZ, J. A.; VOLPE, C. A.; PEREIRA, G. T. Estudo da precipitação máxima em Jaboticabal (SP) pela distribuição de Gumbel utilizando dois métodos de estimação dos parâmetros. Revista Brasileira de Agrometeorologia, Santa Maria, v. 11, n. 1, p. 141-147, 2003.

BELTRAME, L. F. S.; LANA, A. E. L.; LOUZADA, J. A. S. Chuvas Intensas. Porto Alegre: IPH-UFRGS, 1991. 69 p.

BRASIL. Ministério de Agricultura. Normais Climatológicas (1961- 1990). Rio de Janeiro: Ministério da Agricultura, 1992. 84 p.

CAMPOS, H. de. Estatística experimental nãoparamétrica. 3. ed. Piracicaba: ESALQ, 1979. 343 p.

ELTZ, F. L. P.; REICHERT, J. M.; CASSOL, E. A. Período de retorno de chuvas em Santa Maria. Revista Brasileira de Ciência do Solo, Campinas, v. 16, p. 265-269, maio/ago. 1992.

GOMES, F. G.; AQUINO, L. H. de; OLIVEIRA, M. S. de. Estudo da distribuição e freqüência de precipitação pluviométrica máxima em períodos de dez e quinze dias, um mês e um ano, em Lavras (MG) pela distribuição Gumbel. Ciência e Prática, Lavras, v. 13, n. 2, p. 177-184, maio/ago. 1989.

GUMBEL, E. J. Statistics of extremes. New York: Columbia University, 1958. 375 p.

HAAN, C. T. Statistical methods in hidrology. Ames: The Iowa State University, 1977. 377 p.

LEOPOLDO, P. R.; SANSIGOLO, C. A.; MARTINS, D. Análise estatística das intensidades e precipitações máximas de Botucatu. Irrigação e tecnologia moderna, Brasília, v. 16, p. 11-14, mar. 1984.

LOWERY, M. D.; NASH, J. E. A comparison of methods of fitting the double exponential distribution . Journal of Hydrology, Amsterdam, v. 10, n. 3, p. 259275, Feb. 1970.

MANLY, B. F. J. Randomization, Bootstrap and Monte Carlo methods in biology. Boundary Row: Chapman \& Hall, 1998. 399 p.

MOOD, A. M.; GAYBILL, F. A.; BOES, D. C. Introduction to the theory of statistics. 3. ed. New York: J. Wiley \& Sons, 1974. 564 p.

NOBUKUNI, P. Análise das precipitações com duração de 5 minutos a 24 horas, nas regiões de Jaboticabal e Presidente Prudente. 2000. $65 \mathrm{f}$. Dissertação (Mestrado em Agronomia) - Universidade Estadual Paulista, Jaboticabal, 2000.

PFAFSTETTER, O. Chuvas intensas no Brasil. Rio de Janeiro: Ministério da Viação e Obras Públicas, 1957. 420 p.

PINTO, F. R. L. Equações de intensidades-duraçãofreqüência da precipitação para os estados do Rio de Janeiro e Espirito Santo: estimativa e espacialização. 1999. 70 f. Dissertação (Mestrado em Engenharia Agrícola) Universidade Federal de Viçosa, Viçosa, 1999.

SAS INSTITUTE. SAS/IML ${ }^{\circledR}$ Software: usage and reference. Version 6. Cary, 1990. 501 p.

TUCCI, C. M. Hidrologia: ciência e aplicação. Porto Alegre: UFRGS, 2001. 943 p.

VASCONCELloS, S. L. B. de. Análise da quantidade e distribuição pluviométrica na região de Jaboticabal - SP. 1998. 63 f. Dissertação (Mestrado em Agronomia) - Escola Superior de Agricultura Luiz de Queirós, Universidade de São Paulo, Piracicaba, 1998. 
VIANELLO, R. L.; ALVES, A. R. Meteorologia básica e aplicações. Viçosa: UFV, 1991. 449 p.

VIEIRA, S. R.; LOMBARDI NETO, F.; BURROWS, I. T. Mapeamento de chuva diária máxima provável para o Estado de São Paulo. Revista Brasileira de Ciências do Solo, Campinas, v. 15, p. 93-98, jan./abr. 1991.
VIEIRA, D. B.; LOMBARDI NETO, F.; SANTOS, R. P. Análise das intensidades máximas de chuva em Pindorama (SP). Revista Brasileira de Ciências do Solo, Campinas, v. 18, n. 2, p. 255-60, maio/ago. 1994.

VIVANCO, M. J. F. Análise de valores extremos no tratamento estatístico da corrosão de equipamentos. 1994. 107 f. Dissertação (Mestrado em Estatística) Universidade Estadual de Campinas, Campinas, 1994. 